development of supportive ACP-structures in the field of nursing homes $(\mathrm{NH})$. In the region Würzburg (130.000 inhabitants, Bavaria) there is no ACP-program provided. Major purpose of the project is the conceptualization, implementation and evaluation of an effective, target group-specific concept. Within the project needs of nursing home residents (NHR) concerning the process of ACP are reconstructed, barriers of sustainable implementation of ACP are identified.

Methods The study includes problem-centered interviews with NHR $(n=24)$. Maintopics of the interviews: requirements regarding to EoL, communication about needs at EoL, documentation of advance planning, decision-making/-behavior concerning EoLC. Data are analyzed by content analysis.

Results NHR have a lack of knowledge relating to scope, reach and potential objects of advance planning for $\operatorname{EoL}(\mathrm{C})$ even they have already prepared an advance directive or a power of attorny. Often there is no differentiated reflection of own needs even they remark the wish of self-reflection concerning preferences for their EoL. NHR express the wish to compare notes with non-professionals and at eye level, trustful dialogue partners on EoL-topics and decision-making (informal dialogue). Otherwise, the recording of decisions and responsibilities regarding to the EoL takes place within the family-network and in the dialogue with health professionals (formal dialogue).

Conclusion A viable ACP-concept has to close the gap between informal and formal dialogues by creating trustful dialogue spaces and identifying trustful dialogue-partners.

\section{P35 EXPERIENCES WITH APPROACHES TO ADVANCE CARE PLANNING WITH OLDER PEOPLE: A QUALITATIVE STUDY AMONG DUTCH GENERAL PRACTITIONERS}

J Glaudemans*, E Moll van Charante, J Wind, J Oosterink, D Willems. Amsterdam UMC, Amsterdam Public Health research institute, location AMC, Amsterdam, Netherlands

\subsection{6/spcare-2019-ACPICONGRESSABS.119}

Background Advance care planning (ACP) is still used with only a minority of older patients due to a lack of knowledge regarding appropriate approaches to ACP with older people. General practitioners (GPs) may play a key role in ACP with older people. We explored their experiences with different approaches to ACP with older patients in daily practice.

Methods A qualitative study among a purposive sample of 19 Dutch GPs based on semi-structured interviews.

Results Approaches to ACP with older patients can be divided into two categories: systematic and ad-hoc. Systematic approaches consisted of discussing a fixed combination of topics during group information meetings, intakes, comprehensive geriatric assessments, and periodic assessments with community-dwelling older patients who are frail, cognitively impaired, or aged $>75$, and with older patients living in residential care homes. Meetings were aimed at making agreements in anticipation of future care, at providing information and at encouraging older people to take further steps in ACP. With ad-hoc approaches, respondents discussed only one or two topics related to the near future with deteriorating patients or when patients or family-initiated ACP. Systematic and ad-hoc approaches were used simultaneously or sequentially. Due to a lack of time and knowledge respondents seemed to underuse many occasions and topics.
Conclusions Awareness of appropriate systematic and ad-hoc approaches to ACP, and the focus on providing information and encouraging older people to take further steps in ACP can support GPs and improve older patients' access to ACP.

\section{P36 DEVELOPMENT OF THE STADPLAN INTERVENTION ON ADVANCE CARE PLANNING IN CARE DEPENDENT COMMUNITY-DWELLING OLDER PERSONS IN GERMANY}

${ }^{1} \mathrm{~A}$ Kirchner* ${ }^{1} \mathrm{H}$ Langner, ${ }^{1} \mathrm{G}$ Meyer, ${ }^{2} \mathrm{R}$ Schnakenberg, ${ }^{3} \mathrm{~K}$ Silies, ${ }^{4} \mathrm{Y}$ Chuvarayan, ${ }^{2} \mathrm{~F}$ Hoffmann, ${ }^{3} \mathrm{~S}$ Köpke, ${ }^{4} \mathrm{~J}$ Köberlein-Neu, ${ }^{1} \mathrm{~A}$ Berg. ${ }^{1}$ Martin Luther University HalleWittenberg, Halle (Saale), Germany; ${ }^{2}$ Carl von Ossietzky University, Oldenburg, Germany; ${ }^{3}$ University of Lübeck, Lübeck, Germany; ${ }^{4}$ University of Wuppertal, Wuppertal, Germany

\subsection{6/spcare-2019-ACPICONGRESSABS. 120}

Background The STADPLAN project is a multicentre, clusterrandomised controlled trial on advance care planning (ACP) in older people receiving professional home care. The aim of the intervention is to encourage patients to deal with the topic of ACP in a structured way, to nominate a surrogate decision maker and to regularly discuss own preferences and wishes with this person.

Methods Following the MRC framework for complex interventions, we systematically adapted the intervention components of existing ACP programmes to the needs of community nursing care in Germany. The design of the modelled multicomponent intervention was guided by the Behaviour Change Wheel method. Experts reviewed and discussed the proposed intervention programme.

Results The complex intervention addresses patients aged $\geq$ 65 years, nursing professionals and general practitioners comprising:

1. A minimum of two guideline-based conversations led by qualified nurses to be offered to dyads of patients and relatives.

2. A two-day training course including practical exercises to prepare nurses for the conversations.

3. Written information about ACP provided to patients, aiming to encourage patients to reflect on and write down own wishes and health care preferences in various situations. The information offers further local counselling options for the preparation of ACP documents.

4. Participants' general practitioners will receive concise written information about the study.

Conclusion The acceptance, feasibility, and comprehensibility of the complex intervention are currently piloted in four home care services including 120 patients. Results are expected in early 2019 and will be used to optimise the intervention before the efficacy trial.

\section{P37 DIFFERENCES IN THE PERCEPTIONS OF END-OF-LIFE CARE PREFERENCES BETWEEN NON-DYAD PATIENTS AND PROXIES IN AN ASIAN CONTEXT}

EJ Koh, CC Yu*, JA Low. Geriatric Education and Research Institute, Singapore, Singapore

\subsection{6/spcare-2019-ACPICONGRESSABS.121}

Background In Singapore, Advanced Care Planning (ACP) for patients who lack mental capacity is often conducted with the patient's family or proxy. The mismatch of perspectives 